\title{
Magnetic alignment of carbon nanofibers in polymer composites and anisotropy of mechanical properties
}

\author{
Donglu Shi ${ }^{\mathrm{a})}$ and Peng $\mathrm{He}$ \\ Department of Chemical and Materials Engineering, and Department of Mechanical Engineering, \\ University of Cincinnati, Cincinnati, Ohio 45221 \\ Jie Lian \\ Department of Geological Sciences and Department of Materials Science and Engineering, University of \\ Michigan, Ann Arbor, Michigan 48109 \\ Xavier Chaud \\ Consortium de Recherches pour l'Emergence de Technologies Avancées, CNRS, BP 166, \\ F-38042 Grenoble Cedex 9, France \\ Sergey L. Bud'ko \\ Ames Laboratory and Department of Physics and Astronomy, Iowa State University, Ames, Iowa 50011
}

Eric Beaugnon

Consortium de Recherches pour l'Emergence de Technologies Avancées, CNRS, BP 166, F-38042 Grenoble Cedex 9, France

L. M. Wang and Rodney C. Ewing

Department of Geological Sciences and Department of Materials Science and Engineering, University of Michigan, Ann Arbor, Michigan 48109

Robert Tournier

Consortium de Recherches pour l'Emergence de Technologies Avancées, CNRS, BP 166, F-38042 Grenoble Cedex 9, France

(Received 27 May 2004; accepted 4 January 2005; published online 11 March 2005)

\begin{abstract}
Engineering applications of carbon nanofibers and nanotubes require their alignment in specific directions. Single-walled carbon nanotubes can be aligned in a magnetic field due to the presence of small amounts of catalyst elements, such as $\mathrm{Ni}$ and Co. However, for carbon nanofibers, their extremely low magnetic susceptibility is not sufficient for magnetically induced alignment. We present a method of solution-coating of $\mathrm{NiO}$ and $\mathrm{CoO}$ onto the surface of the carbon nanofibers. Due to the NiO- and CoO-coating, these nanofibers can be well aligned in the polymer composites under moderate magnetic field $(3 \mathrm{~T})$. Both transmission electron microscopy and scanning electron microscopy results show the well-aligned nanofibers in a polymer matrix. Mechanical testing shows a pronounced anisotropy in tensile strength in directions normal (12.1 MPa) and parallel (22 MPa) to the applied field, resulting from the well-aligned nanofibers in the polymer matrix. The mechanism of magnetic alignment due to coating of $\mathrm{NiO}$ and $\mathrm{CoO}$ on the nanofiber surface is discussed. () 2005 American Institute of Physics. [DOI: 10.1063/1.1861143]
\end{abstract}

\section{INTRODUCTION}

Recently, an extensive research effort has been devoted to optimizing the properties of carbon nanotubes/nanofibers in polymer composites. ${ }^{1-10}$ For both a fundamental understanding of the properties of nanotubes and engineering applications, it is often necessary to fabricate the nanotubes and nanofibers so that they are aligned in specific directions. Previous researchers have mainly focused their efforts on singlewalled carbon nanotubes due to their considerable magnetic susceptibility that results from the presence of catalyst elements, such as nickel and cobalt. ${ }^{11-14}$ For carbon nanofibers, there have not been any results reported from magnetic alignment as a result of nonmagnetic properties of these materials. Attempts have been made to align nanotubes in polymers by mechanical straining. ${ }^{15}$ However, only partial align-

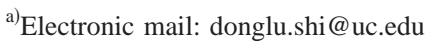

ment was achieved on the surfaces of the sample. Furthermore, the volume fraction of nanotube addition is high, reaching a maximum of $50 \mathrm{wt} \%$. At low concentrations of nanotubes, the anisotropic behavior is at a minimum or unobservable. For mechanical property enhancement in polymer composite materials, the reported nanotube concentration is only between 1 and $5 \mathrm{wt} \% .^{7-10}$ Therefore, it is of great interest to develop magnetic techniques to align a small fraction of nanofibers or multiwalled carbon nanotubes (MWCNT) in polymer composites.

In nanofiber reinforced polymer composites, there are two key issues: (1) the small fraction of nanofibers in the composite matrix must be well dispersed. This issue has been recently dealt with by the authors using a plasma polymerization method; ${ }^{16-21}$ (2) the nanofibers must be oriented along a specified direction by which the mechanical and other physical properties can be maximized. The alignment of the nanotubes and nanofibers must be distributed uni- 
formly throughout the matrix of the composite material. Any gradient in nanotube concentration in the matrix may cause unacceptable variations in the transport and bulk properties. Due to extremely low magnetic susceptibility of carbon nanofibers $\left(\sim 10^{-6} \mathrm{emu}\right)$, the dipole moments induced in the magnetic field are insufficient to cause them to rotate in a viscous liquid and align themselves along the direction of the applied field, even at a high field strength. Therefore approaches are needed to magnetically align the nanofibers in bulk polymer composites.

We present experimental data on the magnetically aligned carbon nanofibers in polystyrene composites. As a result of nickel and cobalt solution-coating on the surfaces of carbon nanofibers, the carbon fibers in magnetic field were aligned. We also report the microstructure, magnetization, and mechanical properties of a polymer composite impregnated with coated carbon nanofibers.

\section{EXPERIMENTAL DETAILS}

Commercial grade Pyrograf III carbon nanofibers were used as substrates. ${ }^{22}$ The Pyrograf III nanofibers were 70 $200 \mathrm{~nm}$ in diameter, several microns in length. The surfaces of the nanofibers were treated by a plasma polymerization method. The plasma reactor used for thin film deposition on the nanofibers and the associated processing conditions have been previously presented. ${ }^{17,18}$

For magnetic alignment, the nanofibers were coated with nickel and cobalt oxides by a solution method. Five grams of $\mathrm{Ni}\left(\mathrm{NO}_{3}\right)_{2} \cdot 6 \mathrm{H}_{2} \mathrm{O}$ and $\mathrm{Co}\left(\mathrm{NO}_{3}\right)_{2} \cdot 6 \mathrm{H}_{2} \mathrm{O}(2.5 \mathrm{~g}$ each $)$ were dissolved in $100 \mathrm{ml}$ water. One gram of nanofibers was then mixed with the solution by ultrasonic means for $2 \mathrm{~h}$. Finally, the mixed solution was dried at $100{ }^{\circ} \mathrm{C}$.

The nanofiber concentration for this study was 3 wt $\%$. Before magnetic alignment, $2 \mathrm{~g}$ of polystyrene were dissolved in $30 \mathrm{ml}$ toluene to make the solution. Meanwhile, $\mathrm{NiO} / \mathrm{CoO}$-coated nanofibers were dispersed into $30 \mathrm{ml}$ toluene by ultrasonic means for $2 \mathrm{~h}$. The $\mathrm{NiO} / \mathrm{CoO}$-coated nanofibers were also plasma coated with polystyrene for enhanced dispersion. This processing procedure suggests the presence of a second layer coating on the surface of the nanofiber. Then these two solutions were ultrasonically mixed together for another $2 \mathrm{~h}$. Finally, the solution was poured into the mold, ready for magnetic alignment.

The system was specially designed for creating a horizontal center field up to $5 \mathrm{~T}$ with an Oxford Instruments Superconducting Magnet as shown in Fig. 1. The system was based on a split pair coil mounted in a cryostat. The design provided a room temperature access to the field in two 120mm-diam bores, one horizontally aligned on the field axis and one vertically through the magnet split. The NbTi coil operated in a liquid helium bath at $4.2 \mathrm{~K}$ and generated a horizontal magnetic field up to $5 \mathrm{~T}$. The given field homogeneity was 1 part in $10^{3}$ over a 10 -mm-diam spherical volume.

The magnetic alignment was conducted at room temperature. The field was first ramped up to a given value, such as $1 \mathrm{~T}$, and stabilized. The mold containing the liquid of nanofiber and polystyrene mixture was then placed at the center region where the magnetic field reached the maximum

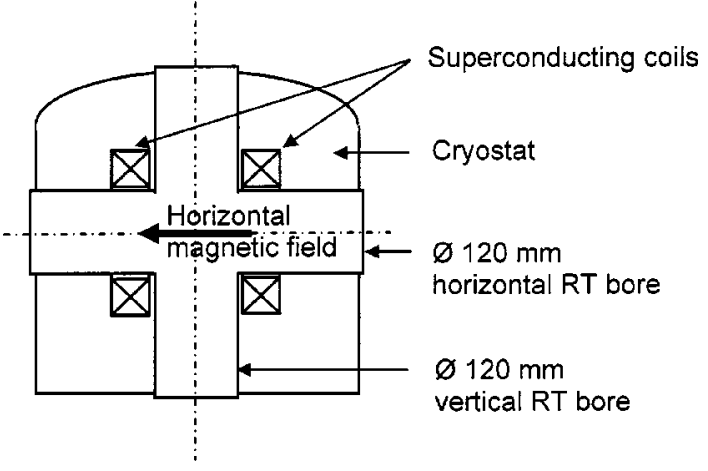

FIG. 1. Schematic diagram showing the magnet used for nanofiber alignment.

value $(1,2$, and $3 \mathrm{~T})$. Since it was a horizontal field, the direction of the field was parallel to the surface of the liquid. As a result of the interaction between the applied field and the magnetic moments of coated $\mathrm{NiO} / \mathrm{CoO}$ on the nanofibers, they rotated in the viscous liquid and aligned themselves with the direction of the field. At same time, the solvent was being slowly evaporated through the opening at the top of the magnet. The magnetic field was maintained for at least $24 \mathrm{~h}$ for a complete consolidation of the polymer composite. After the sample was dried, it was removed from the mold for microstructure and property characterization.

The microstructure and fracture morphology of both coated and uncoated nanofiber composites were characterized by scanning electron microscopy (SEM) observation using a Philips XL30 FEG SEM. Transmission electron microscopy (TEM) images were acquired using a JEOL 2010F TEM to show the interface structures that are responsible for the improved properties. TEM samples of coated nanofibers were prepared by dispersing the nanofibers directly on holycarbon films supported with $\mathrm{Cu}$ grids. TEM samples of the composite samples were prepared by ultramicrotomy with a cutting thickness of $60 \mathrm{~nm}$. An Instron mechanical testing machine, model 2525-818, with a $1 \mathrm{~mm} / \mathrm{min}$ cross head speed was used for the tensile test.

Direct current magnetization measurements were performed in Quantum Design MPMS-5 superconducting quan-

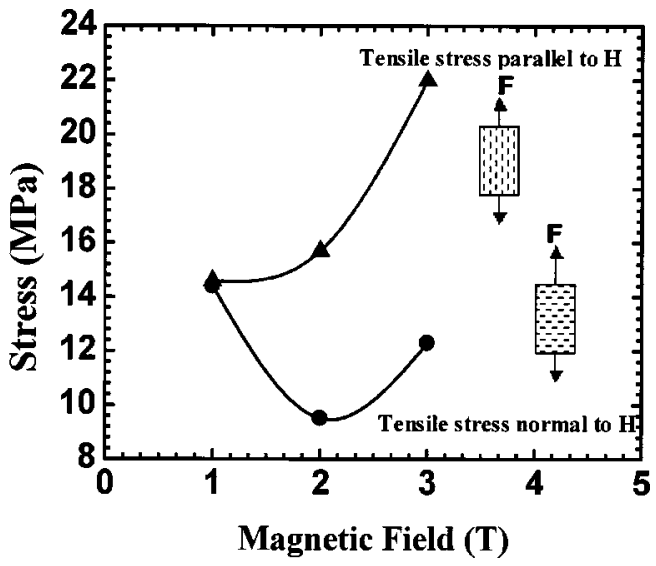

FIG. 2. Tensile stress vs applied magnetic field for the nanofiber polystyrene samples oriented with the tensile force normal and parallel to the direction of the field. 

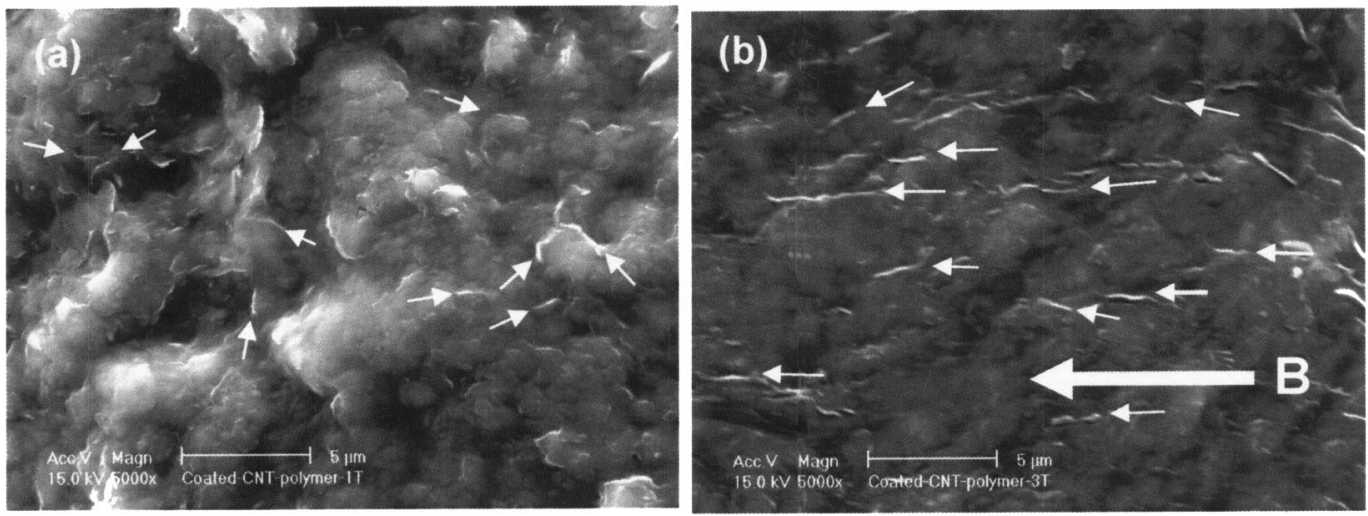

FIG. 3. SEM micrographs showing surface of the sample that was magnetically aligned at $1 \mathrm{~T}$ (a) and aligned at $3 \mathrm{~T}$ (b).

tum interference device (SQUID) magnetometers. Samples were cut in the shape of squares (to avoid demagnetization factor considerations) with the edges parallel and perpendicular to the alignment field direction. Temperaturedependent measurements were performed in a $5 \mathrm{~T}$ applied field. Field-dependent magnetization was measured at $300 \mathrm{~K}$.

\section{RESULTS AND DISCUSSION}

The nanofibers were first liquid-coated with $\mathrm{NiO} / \mathrm{CoO}$, then plasma-coated with polystyrene. The nanofibers (3 wt \% of double-layer-coated: first layer $\mathrm{NiO} / \mathrm{CoO}$ and second layer polystyrene) were magnetically aligned in the polystyrene matrix to form a composite by the previously described procedure. After magnetic alignment and consolidation, a dried sample was sectioned into several pieces of $40 \times 6$ $\times 0.4 \mathrm{~mm}^{3}$ dimensions. To determine the anisotropy in mechanical properties, these samples were cut in such a way that the tensile test could be performed from two directions, i.e., tensile stresses parallel and perpendicular to the direction of the applied magnetic field. Figure 2 shows the tensile strength as a function of the applied magnetic field for both directions. There is a pronounced anisotropy evident from the two stress configurations. At $1 \mathrm{~T}$, the tensile stresses for both the parallel and perpendicular directions show minimum differences. However, this difference has increased from 9.45 to $15.69 \mathrm{MPa}$ at $2 \mathrm{~T}$, indicating a large anisotropy. At even higher field of $3 \mathrm{~T}$, the tensile strength has increased from 12.10 to $22 \mathrm{MPa}$, a nearly $90 \%$ increase in mechanical strength. Although there is a sample dependence on mechanical properties, the anisotropy increase is consistent with the changes in the field strength.

Figure 3 shows the SEM surface microstructure of the samples magnetically aligned at $1 \mathrm{~T}[\mathrm{Fig} .3(\mathrm{a})]$ and $3 \mathrm{~T}[\mathrm{Fig}$. 3(b)]. At low magnification, the nanofibers can be seen in a random arrangement in the $1 \mathrm{~T}$ sample, suggesting that the field strength at $1 \mathrm{~T}$ is insufficient to orient the nanofibers along the field direction. However, at $3 \mathrm{~T}$, most of the nanofibers are well aligned, although some of them are not exactly in line with the main magnetic field direction. Figure 4 shows the TEM images of the 1 and $3 \mathrm{~T}$ samples. Due to high magnification, it is difficult to see a large number of nanofibers within the field of view of the TEM. Therefore, several images were taken from adjacent areas and are combined. Figures 4(a) and 4(b) are images from the $1 \mathrm{~T}$ sample; although a few nanofibers can be seen, the random nature is evident in these images. In Figs. 4(c)-4(e), three images from the $3 \mathrm{~T}$ sample are combined showing the alignment of the nanofibers. The nanofibers are seen in these images as the translucent rods that are indicated by small arrows on both sides.

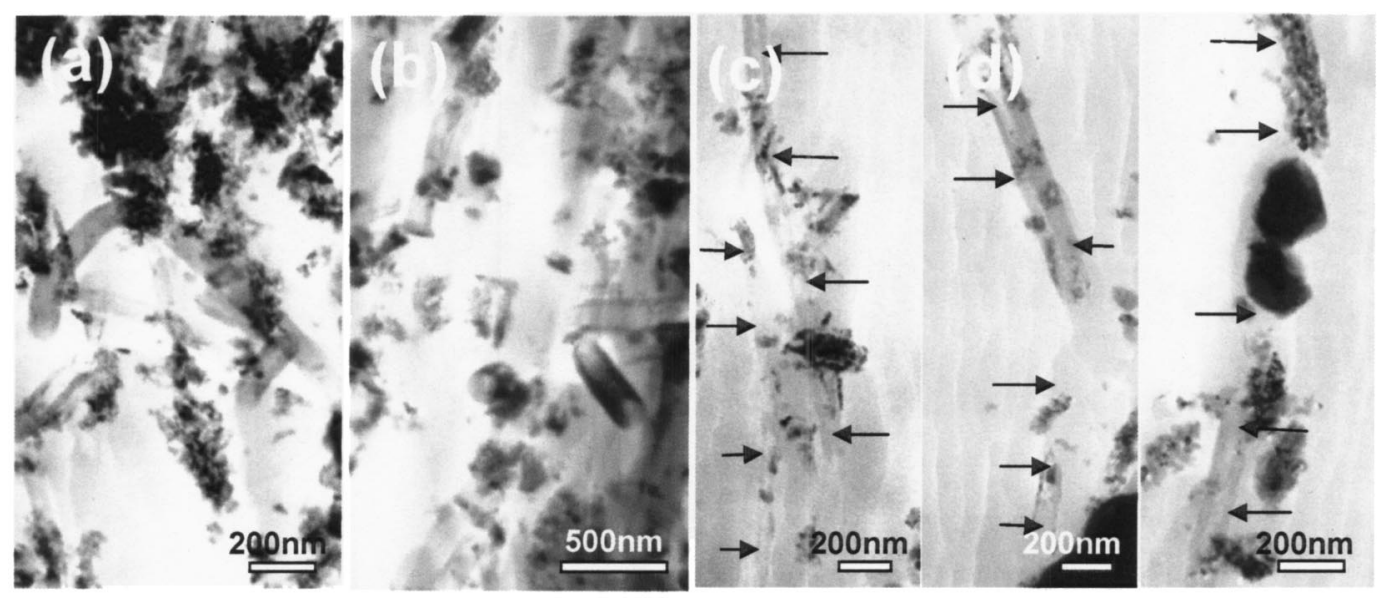

FIG. 4. TEM images showing (a) and (b) the randomly oriented nanofibers in the $1 \mathrm{~T}$ sample; (c)-(e) the aligned nanofibers in the $3 \mathrm{~T}$ sample. The translucent rods are nanofibers indicated by the small arrows. The dark spots on the nanofiber surfaces are $\mathrm{NiO} / \mathrm{CoO}$ coatings. 


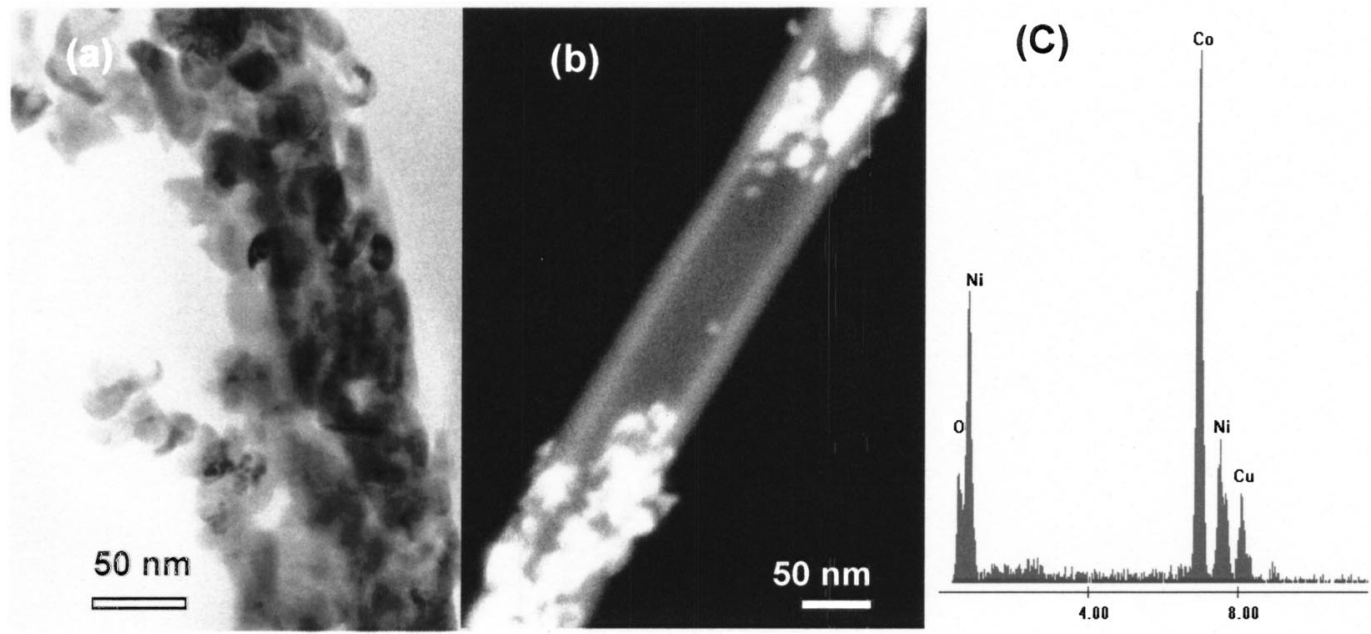

FIG. 5. (a) Bright-field TEM images of the surface coating of $\mathrm{NiO} / \mathrm{CoO}$; (b) Z-contrast image showing the partially coated feature of NiO/CoO on the surfaces of the nanofibers. $\mathrm{NiO}$ and $\mathrm{CoO}$ particles show bright contrast due to the relative heavier element mass $Z$ as compared with that of carbon nanofibers; and (c) EDS spectrum of the $\mathrm{NiO} / \mathrm{CoO}$ coating on nanofibers.

Figure 5 shows the TEM images of the surface $\mathrm{NiO} / \mathrm{CoO}$ coating on the nanofibers for the $3 \mathrm{~T}$ sample. In Fig. 5(a), the $\mathrm{NiO} / \mathrm{CoO}$ coating on the surfaces of the nanofibers is seen, as well as that of crystallized particles. However, the coating is not entirely covering the surfaces of some of the nanofibers as shown in Fig. 5(b). EDS measurement of particles showing bright contrast indicated a high concentration of $\mathrm{Ni}$ and $\mathrm{Co}$ within the coating region [Fig. $5(\mathrm{c})]$.

The magnetization of the nanofiber composite was measured using a Quantum Designed SQUID. The sample was sectioned into a $5 \times 5 \mathrm{~mm}$ square (same thickness) with each axis parallel and normal to the direction of the alignment. Figure 6 shows the temperature dependence of magnetization

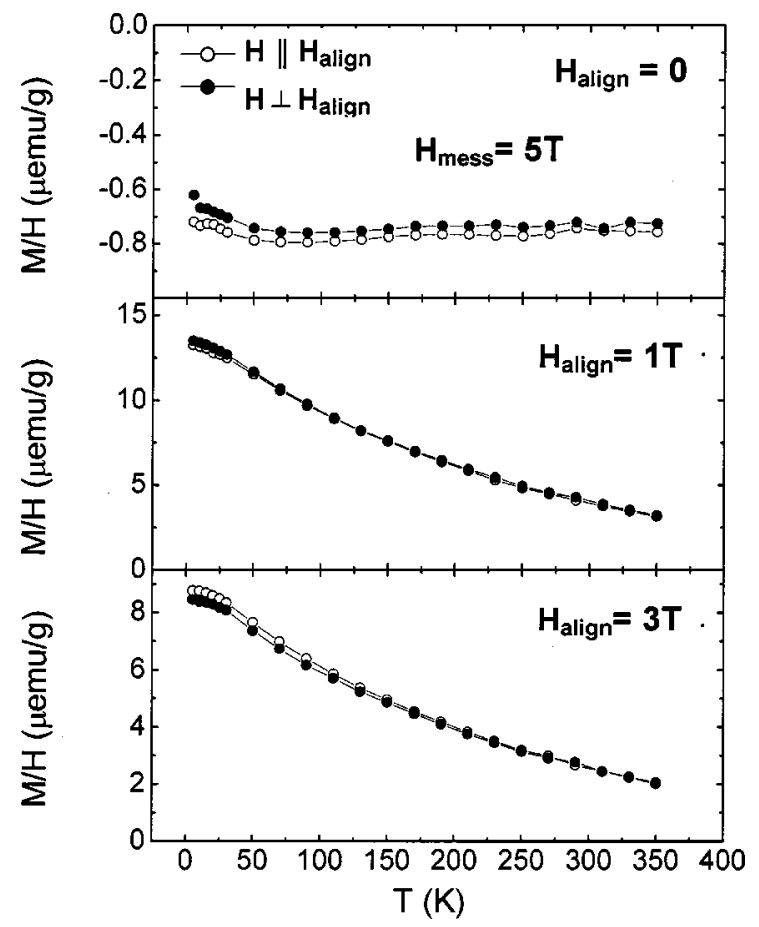

FIG. 6. Magnetization vs temperature for samples aligned at 0,1 , and $3 \mathrm{~T}$. for the samples aligned at 0,1 , and $3 \mathrm{~T}$ in both directions. The magnetization curves do not exhibit pronounced differences for the applied field parallel and perpendicular to the direction of the alignment (Fig. 6). However, the magnitude of magnetization showed a considerable decrease as the temperature increased from 4 to $350 \mathrm{~K}$ for the 1 and $3 \mathrm{~T}$ samples; while it remained more or less unchanged for the 0 $\mathrm{T}$ sample. Only a slight difference was observed between the two configurations for the unaligned sample.

The main effort of this study was to determine whether it was possible to magnetically align nanofibers that have a low magnetic susceptibility. According to the literature, the magnetic susceptibility of the various forms of carbon has been measured, including diamond, graphite, $\mathrm{C}_{60}$, and nanotubes. Carbon nanotubes exhibit a diamagnetic susceptibility, on the order of $10^{-6} \mathrm{emu} / \mathrm{g}$ at $300 \mathrm{~K}^{13}$ In previous studies, the magnetic alignment of MWCNT in polyester composite was completed at extremely high field strength of up to $10 \mathrm{~T} .{ }^{13} \mathrm{In}$ these studies, magnetic impurities were believed to contribute to the considerable level of magnetic susceptibility of the nanotubes. For nanofibers, due to $\mathrm{NiO} / \mathrm{CoO}$ coating, the magnetic moments were significantly increased $(8.5 \mathrm{emu} / \mathrm{g})$ as indicated in the SQUID magnetization measurements. Therefore, the interaction between the applied field and induced moments resulted in the alignment of nanofibers along the direction of the field.

It should be noted that the $\mathrm{NiO} / \mathrm{CoO}$ coating is seen in Fig. 5 to be not uniform. For the purpose of magnetic alignment, in fact, it need not to be uniform as the $\mathrm{NiO} / \mathrm{CoO}$ particles are potent magnetic materials. As long as these particles can induce enough moments for magnetic alignment, the uniformity of the coating is not necessary. Furthermore, the roughness of the coating can also contribute to sufficient interface interaction with the matrix for the enhancement of mechanical properties.

Assuming that each $\mathrm{NiO}$ or $\mathrm{CoO}$ particle has an average moment $\langle\mu\rangle$ and each nanofiber has been uniformly coated with these "particles," then the moment of these nanotubes has a mean value: $\langle\mathbf{M}\rangle=N\langle\mu\rangle$, where $N$ is the number of 
particles and $\langle\mathbf{M}\rangle$ is the moment along the long axis of the nanofiber. ${ }^{23}$ The aspect ratio is: $\rho=r / l$, where $r$ and $l$ are the radius and the length of the nanofiber, respectively. When $\rho<\rho_{c}$,

$$
\{\langle\mathbf{M}\rangle / r l\}\langle\mu\rangle=\text { const. }
$$

However, when $\rho>\rho_{c}$,

$$
\{\langle\mathbf{M}\rangle / r l\}\langle\mu\rangle=\text { decreasing function of } \rho .
$$

According to the SEM results, the length of the nanofiber is on the order of $2 \mu \mathrm{m}$, and diameter of $100 \mathrm{~nm}$, giving a $\rho$ value of 0.05 . This aspect ratio should result in a pronounced behavior of alignment under a significant applied field. As can be seen in Fig. 6, the sample that is not subjected to the magnetic field exhibits rather low magnetization on the order of $0.7 \mathrm{emu} / \mathrm{g}$. This is due to the random distribution of the nanofibers with the $\mathrm{NiO} / \mathrm{CoO}$ coatings. The magnetic moments induced from each nanofiber could cancel each other, leading to a small net moment. However, as the sample is subjected to externally applied field, the nanofibers assume a preferred orientation along the direction of the field. Therefore, the net moments are significantly increased for the 1 and $3 \mathrm{~T}$ samples between 4 and $350 \mathrm{~K}$. As a result of the nanoscale dimensions of these fibers, the magnetization does not exhibit noticeable difference for the fields normal and parallel to the direction of alignment.

In the viscous liquid, the torque induced by the applied field on each nanofiber can be calculated based on the magnetic moment induced from the $\mathrm{NiO} / \mathrm{CoO}$ coating. However, the $\mathrm{NiO} / \mathrm{CoO}$ coating was observed not to be uniform on every nanofiber, therefore it is difficult to determine the precise values of the torque. Nonetheless, an average value of individual moment $\langle\mu\rangle$ maybe estimated if the coating geometry is known. We may conveniently assume a core-shell structure based on the geometry of the nanofibers. To be more accurate on this assumption, a better and more uniform coating is being developed in our laboratory. On the other hand, the rotational dynamics of the nanotubes in the viscous liquid is also important in order to understand the mechanisms of the alignment. In this experiment, the effort was only focused on determining the distribution and the degree of nanofiber alignment in the polymer composite. The factors that affect the nanofiber rotational dynamics include the nanofiber aspect ratio, coating thickness and uniformity, and the strength of the applied field. Further research is under way in order to study the effects of these factors on magnetic alignment of carbon fibers and nanotubes.

In summary, an approach has been developed to magnetically align the carbon nanofibers in polymer composites. As a result of $\mathrm{NiO} / \mathrm{CoO}$ coating, carbon nanofibers can be magnetically induced to rotate in viscous polymer liquid. Both TEM and SEM results have shown clear evidence of magnetic alignment. Due to strong magnetic moments induced from the $\mathrm{NiO} / \mathrm{CoO}$ coatings on the nanofibers, only a moderate applied field ( $3 \mathrm{~T})$ is sufficient to rotate the nanofibers in the viscous liquid. The mechanical tensile testing results show pronounced anisotropy which is consistent with the aligned nanofiber microstructure observed. Such a method can be applied to other nano- and micro-species for various composite materials. Therefore, the method can be used in any general alignment of nonmagnetic materials.

\section{ACKNOWLEDGMENTS}

The authors are grateful to Mark Schulz for providing nanofibers in this research. The TEM and SEM analyses were conducted at the Electron Microbeam Analysis Laboratory at the University of Michigan, and supported by the Office of Basic Energy Sciences of the U.S. Department of Energy under Grant No. DE-FG02-97ER45656. This research was supported in part by a grant from Ohio Aerospace Institute. The magnetization measurements were conducted at Ames Laboratory, and supported by the Director for Energy Research, Office of Basic Energy Sciences, under Contract No. W-7405-Eng-82.

${ }^{1}$ S. Iijima, Nature (London) 354, 56 (1991).

${ }^{2}$ R. H. Baughman, C. Cui, A. A. Zakhidov, Z. Iqbal, J. N. Barisci, G. M. Spinks, G. G. Wallace, A. Mazzoldi, D. De Rossi, A. G. Rinzler, O. Jaschinski, S. Roth, and M. Kertesz, Science 284, 1340 (1999).

${ }^{3}$ M. Gao, L. Dai, R. H. Baughman, G. M. Spinks, and G. G. Wallace, Proc. SPIE 3987, 18 (2000).

${ }^{4}$ V. G. Hadjiev, M. N. Iliev, S. Arepalli, P. Nikolaev, and B. S. Files, Appl. Phys. Lett. 78, 3193 (2001).

${ }^{5}$ C. Liu, H. M. Cheng, H. T. Cong, F. Li, G. Su, B. L. Zhou, and M. S. Dresselhaus, Adv. Mater. (Weinheim, Ger.) 12, 1190 (2000).

${ }^{6}$ D. A. Walters, M. J. Casavant, X. C. Qin, C. B. Huffman, P. J. Boul, L. M. Ericson, E. H. Haroz, M. J. O'Connell, K. Smith, D. T. Colbert, and R. E. Smalley, Chem. Phys. Lett. 338, 14 (2001).

${ }^{7}$ J. Zhu, J. D. Kim, H. Q. Peng, J. L. Margrave, V. N. Khabashesku, and E.

V. Barrera, Nano Lett. 3, 1107 (2003)

${ }^{8}$ E. T. Tostenson and T. W. Chou, J. Phys. D 35, L-77 (2002).

${ }^{9}$ M. Cadek, J. N. Coleman, V. Barron, K. Hedicke, and W. J. Blau, Appl. Phys. Lett. 81, 5123 (2002).

${ }^{10}$ S. J. V. Frankland, V. M. Harik, G. M. Odegard, D. W. Brenner, and T. S. Gates, Compos. Sci. Technol. 63, 1655 (2003).

${ }^{11}$ H. Garmestani, M. S. Al-Haik, K. Dahmen, R. Tannenbaum, D. Li, S. S. Sablin, and M. Y. Hussaini, Adv. Mater. (Weinheim, Ger.) 15, 1918 (2003).

${ }^{12}$ E. S. Choi, J. S. Brooks, D. L. Eaton, M. S. Al-Haik, M. Y. Hussaini, H. Garmestani, D. Li, and K. Dahmen, J. Appl. Phys. 94, 6034 (2003).

${ }^{13}$ T. Kimura, H. Ago, M. Tobita, S. Ohshima, M. Kyotani, and M. Yomura, Adv. Mater. (Weinheim, Ger.) 14, 1380 (2002).

${ }^{14}$ J. E. Fischer, W. Zhou, J. Vavro, M. C. Llaguno, C. Guthy, R. Haggenmueller, M. J. Casavant, D. E. Walters, and R. E. Smalley, J. Appl. Phys. 93, 2157 (2003)

${ }^{15}$ F. Tsui, L. Jin, and O. Zhou, Appl. Phys. Lett. 76, 1452 (2000).

${ }^{16}$ W. J. van Ooij, S. Eufinger, and T. H. Ridgway, Plasmas Polym. 1, 231 (1996).

${ }^{17}$ D. Shi, J. Lian, P. He, L. M. Wang, W. J. Van Ooij, M. Schulz, Y. J. Liu, and D. B. Mast, Appl. Phys. Lett. 81, 5216 (2002).

${ }^{18}$ D. Shi, J. Lian, P. He, L. M. Wang, M. Schultz, F. Xiao, L. Yang, and D. Mast, Appl. Phys. Lett. 83, 5301 (2003).

${ }^{19}$ D. Shi, S. X. Wang, W. J. v. Ooij, L. M. Wang, J. G. Zhao, and Z. Yu, Appl. Phys. Lett. 78, 1243 (2001).

${ }^{20}$ D. Shi, S. X. Wang, W. J. van Ooij, L. M. Wang, J. G. Zhao, and Z. Yu, J. Mater. Res. 17, 981 (2002).

${ }^{21}$ D. Shi, P. He, J. Lian, L. M. Wang, and W. J. van Ooij, J. Mater. Res. 17, 2555 (2002)

${ }^{22}$ Applied Sciences Inc., www.apsci.com

${ }^{23}$ D. Halliday and R. Resnick, Fundamentals of Physics, 2nd ed. (Wiley, New York, 1981), p. 539. 